\title{
Efficient Implementation of a Multi-tone - Sine - Generation Method for Electrical Impedance Spectroscopy Sensors
}

Alexander Renner ${ }^{{ }_{1}, 2}$, Manfred Herz ${ }^{{ }_{1}}$

${ }^{*} \mathrm{i}$ C-Haus $\mathrm{GmbH}$, Am Kuemmerling 18, 55294 Bodenheim, Germany

${ }^{* 2}$ TU Kaiserslautern,Lehrstuhl ISE / EIT,Erwin-Schrödinger-Straße,67663 Kaiserslautern, Germany

\section{Summary}

This paper presents an architectural study and first implementation of a measurement system for impedance spectroscopy. Impedance spectroscopy has shown an increasing potential in quite heterogeneous tasks, including inspection applications. These require a high throughput, so the goal of this investigation is the potential speed-up by applying multitone instead of a sweep of single sine waves measurement. An architecture has been conceived and a first implementation on PCB and desktop equipment level has been achieved. First experiments showed a bandwidth up to $90 \mathrm{MHz}$ of the system.

\section{Introduction}

Impedance Spectroscopy and related methods established themselves in the sensor measurement and instrumentation community. Applications are ranging from analysis of chemical substances, chemical processes or bio-medical applications to characterization of materials. The constraints for impedance spectroscopy (IS) are very different for these sensor applications. The system has to be optimized for measurement speed, impedance frequency, AC/DC current, voltage range, equipment cost and size.

In time-critical measurement applications e.g. characterization of dynamic biologic systems, multi-tone test-signals are used to reduce the measurement time [1],[2]. State-ofthe-art desktop equipment meets the functional needs but are very big and costly. The commercially available integrated system ADUCM350 [5] already provides digital programmable multi-tone (MT) measurement signals for IS, but only in the frequency range $80 \mathrm{~Hz}$

to $75 \mathrm{kHz}$. Other solutions have fixed test signals or are specialized for only one application.

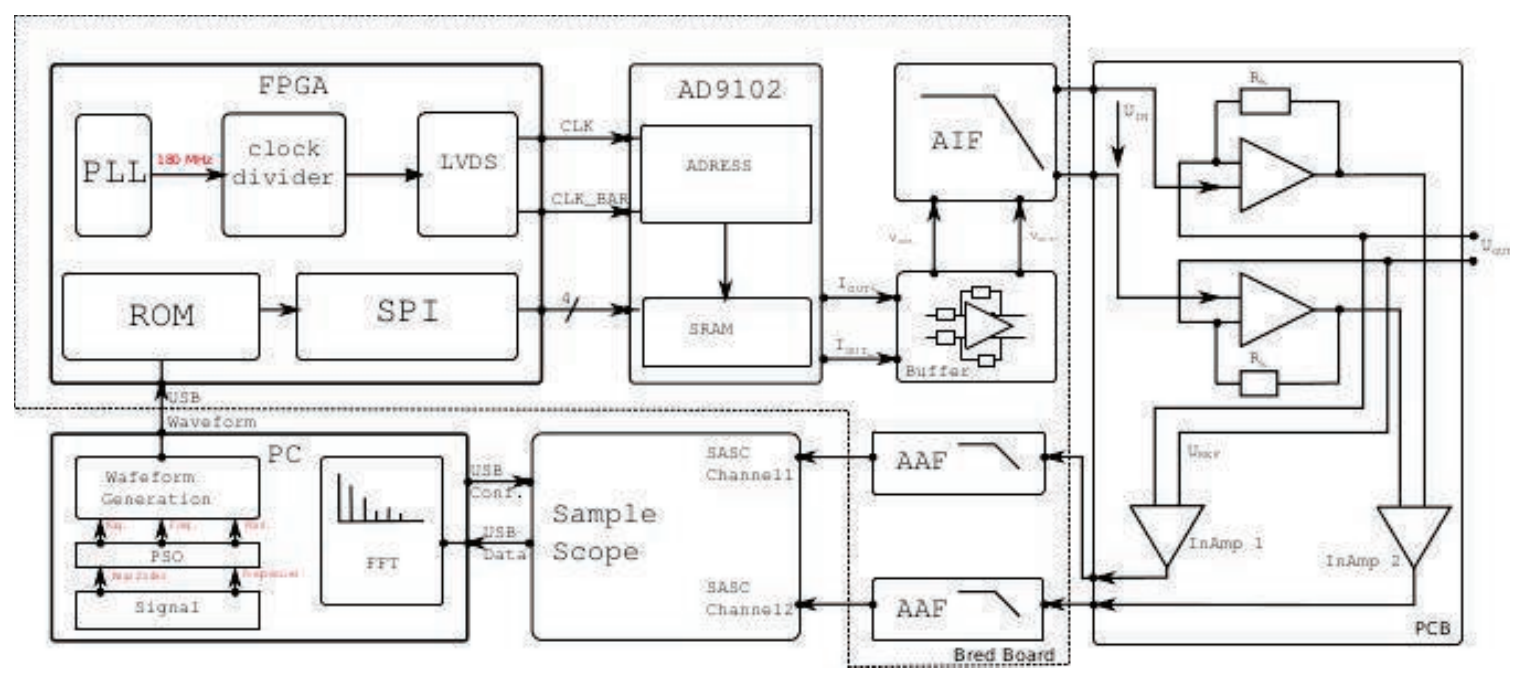

Figure 1: simplified blockdiagramm of the system 


\section{Architecture and Concept}

This work focuses on creating a concept-study for a multi-tone IS-System up to $100 \mathrm{MHz}$. The architecture of the proposed IS-measurement system consists of different design blocks. These blocks are, the optimization of the multi-tone signal by a Particle-Swarm-Optimization. Storing the computed signal-waveform in a SRAM and and apply the stored data to a DAC to generate a MT waveform. The frequency of the multi-tone signal can be varied by changing the controlling clock-rate. For the signal-output / driving stage, a structure with differential non-inverting amplifier's were used. The measured data is sampled with "Sample - Scope" from IC-Haus (ic227). The evaluation of the measured data was done with a Octave and Python script. A simplified block-diagram of the system is showed in Figure 1. The system was built on PCB and bread-boarding level, for testing and giving a proof of the concept. Only on off-the-shell devices were used for the design. The main aim of the proposed system was to understand the possibilities and limitations of the multi-tone concept in discrete or potentially integrated implementation, on the way to creating a multi-tone IS-System up to $100 \mathrm{MHz}$. The main idea behind the architecture of the software part in the systemarchitecture is to have software which fulfills the needs of the multi-tone IS measurement, and can be easily fitted to different measurement scenarios. For this aim, the measurements were controlled by an Octave-script and the measurement results were computed and displayed by a Python-script. These script based languages provide many built in functions to control and evaluate the measurements (e.g. FFT, executing program's, controlling communication, etc.). Another very important part of the system is to evaluate a multi-tone waveform with a good Signal-to-Noise-Ratio ("SNR"). For a good SNR the phases of the discrete frequencies in the multi-tone signal have to be optimized. This will be done with a Particle-Swarm-Optimization ("PSO") approach here. The optimization process and the theory behind, will be discussed in the next section.

\section{Particle swarm optimization for computing Phases}

Most systems are limited in the maximal amplitude of the measurement signal, and added noise. In order to improve the measurement, the power of the signal needs to be maximized for a specific maximal amplitude. The aim is to get the best SNR and add robustness to other additive errors. The Crest Factor ("CF") describes the ratio between the maximum magnitude to the root-mean-square ("rms") of a signal. The CF is in eq. 1 which was used to assess the quality of a signal, and the goal of the optimization was to lower the CR of a signal. The rms was computed with help of the Parsevals's theorem (eq. 2), by knowing the amplitudes in the spectrum of the signal.

$$
\begin{gathered}
C F=\frac{\max [x(t)]}{r m s} \\
r m s=\sqrt{\frac{1}{N^{2}} \sum_{k=0}^{N-1}\left|X[k]^{2}\right|}
\end{gathered}
$$

The optimization was performed by a PSO similar to [4]. The main advantage's of the PSO, compared to other optimization methods, is that there's no restrictions to the signal in the computation. This algorithm is also simple enough to be easily manipulated, and adapted to the needs of the problem. For example by applying conditions for min and max magnitude for a signal.

$$
x(t)=\sum_{i=0}^{N} a_{i} \sin \left(2 \pi f_{0} n_{i} t+\varphi_{i}\right)
$$


In eq. 3 the $a_{i}$ are the Amplitudes, $n_{i}$ the frequencies, $\varphi_{i}$ the Phases, $N$ the Number of frequency's in the signal, and $f_{0}$ the base frequency of the multi-tone signal.

The multi-tone signal will be constructed according to eq. 3. for the computation the basefrequency $f_{0}$ was set to $1 \mathrm{~Hz}$, and was multiplied to set different frequency ranges for the multi-tone signal. The different frequencies will be set with $\mathrm{n}_{\mathrm{i}}$, which is a integer value. Otherwise the resulting multi-tone signal will be non continuous, and leakage effects will appear in the FFT. The $\varphi_{i}$ are the phases for each included frequency which will be optimized by the PSO to get a signal with a low CF. The PSO constructs the multi-tone signal by, the optimization according to the wave-forms of eq. 3 and the cost-function CF of eq. 1 . The PSO optimized signal was compared with other methods, to estimate the quality of the optimization. The comparison showed, in the most cases, a improvement to other optimization algorithm's (e.g. [3]). The comparison with other methods had not always a big improvement, but here the simple algorithm without conditions comes into account, and made the PSO the first choice. For the measurements a signal was computed with all primes from 1 to 100 for frequencies in the signal, as described in [6]. This was done, to avoid disturbances by harmonic due to nonlinear measured impedance. Figure 2 shows such a signal, the red lines are the non optimized signal and the blue lines are for the PSO optimized signal. With knowledge of the measured impedance, e.g. position of the poles, can the signal be modulated to the needs of the measurement, by varying the position and number of frequency-points and magnitudes. It is also possible to apply a DC offset to the signal after the optimization.

\section{Signal Generation}

The idea for the multi-tone measurement-system was to store the computed, and PSO optimized waveform into a RAM. The output signal will be generated with a DAC, in a clocked process. In Figure 3 a simplified block-diagram of the signal-generation stage is shown. For this task the AD9102 from Analog-Devices was chosen. The AD9102 is a TxDAC and waveform generator with a 14 bit DAC, with a digital synthesizer (DDS). A on-chip 4096×14 bit pattern memory allows to store the multi-tone waveform, and the DAC has a maximum sampling rate of 180 MSPS. This allows to generate multi-tone signals up to 90 $\mathrm{MHz}$. For the signal-generation a LVDS-clock is needed, and the programming is done with a SPI interface. For programming and controlling of the AD9102, a PCB with a Cyclone III FPGA was used (see the FPGA block given in Fig. 1). In the FPGA a ROM was initialized, with the programming sequence for the AD9102. For clock-generation a PLL with $180 \mathrm{MHz}$ was initialized. The PLL is controlled by a clock-divider with a LVDS output-stage, for the waveform-generation.
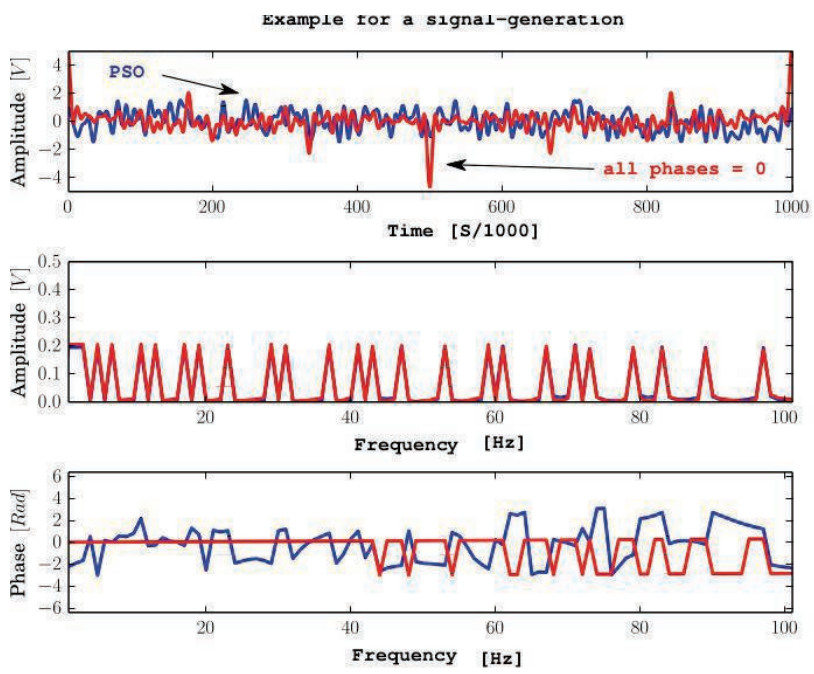

Figure 2: Example for a signal-generation with the PSO.

Signal consists of all primes from 1-100. 
The signal generation part was implemented on a bread-board, this limited the maximum usable frequency to $50 \mathrm{MHz}$. By a frequency above $50 \mathrm{MHz}$ there was to much noise and other parasitic on the signal for practical use, because of the bread-board implementation. In the data-sheet oft the AD9102 it was recommended to use either a transducer or a differential operational amplifier. This is required to compensate differences in the output stage, and to rise the signal to specific common mode voltage.

For this need, a differential op-amp was chosen because he can operate in a wider bandwidth than a transducer (see magenta-framed part with part AD4927 in Fig 3). The AIF in Fig 3 is composed of 3 buffered passive 5 pole LC low-pass-filter. The filtered output can be selected by a block of multiplexers. By varying the bits of the waveform the number of

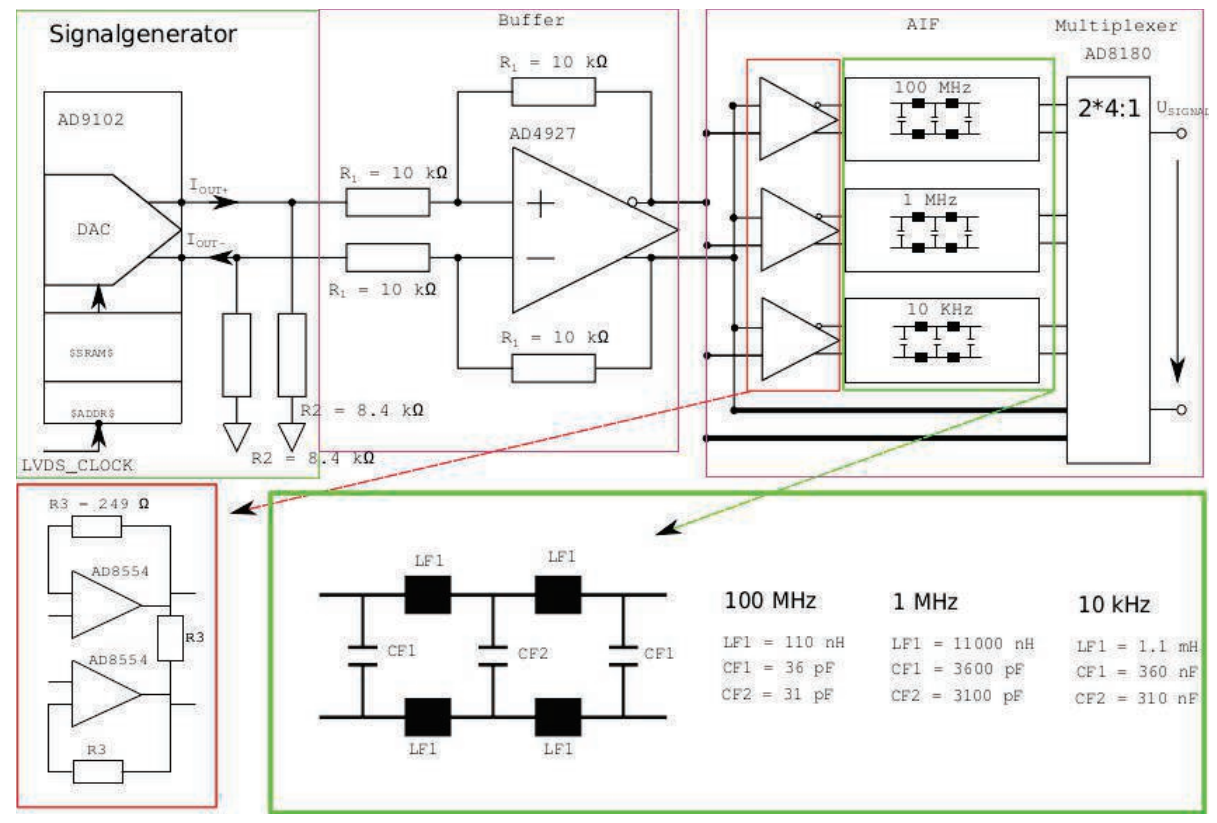

Figure 3: Signal generator

filters was reduced to three for fife decades. This reduced the complexity of the system drastically. The AD9102 is also capable of creating pulsed, and pseudo-random wave-form's, but this was not tested in this work.

\section{Signal Output}

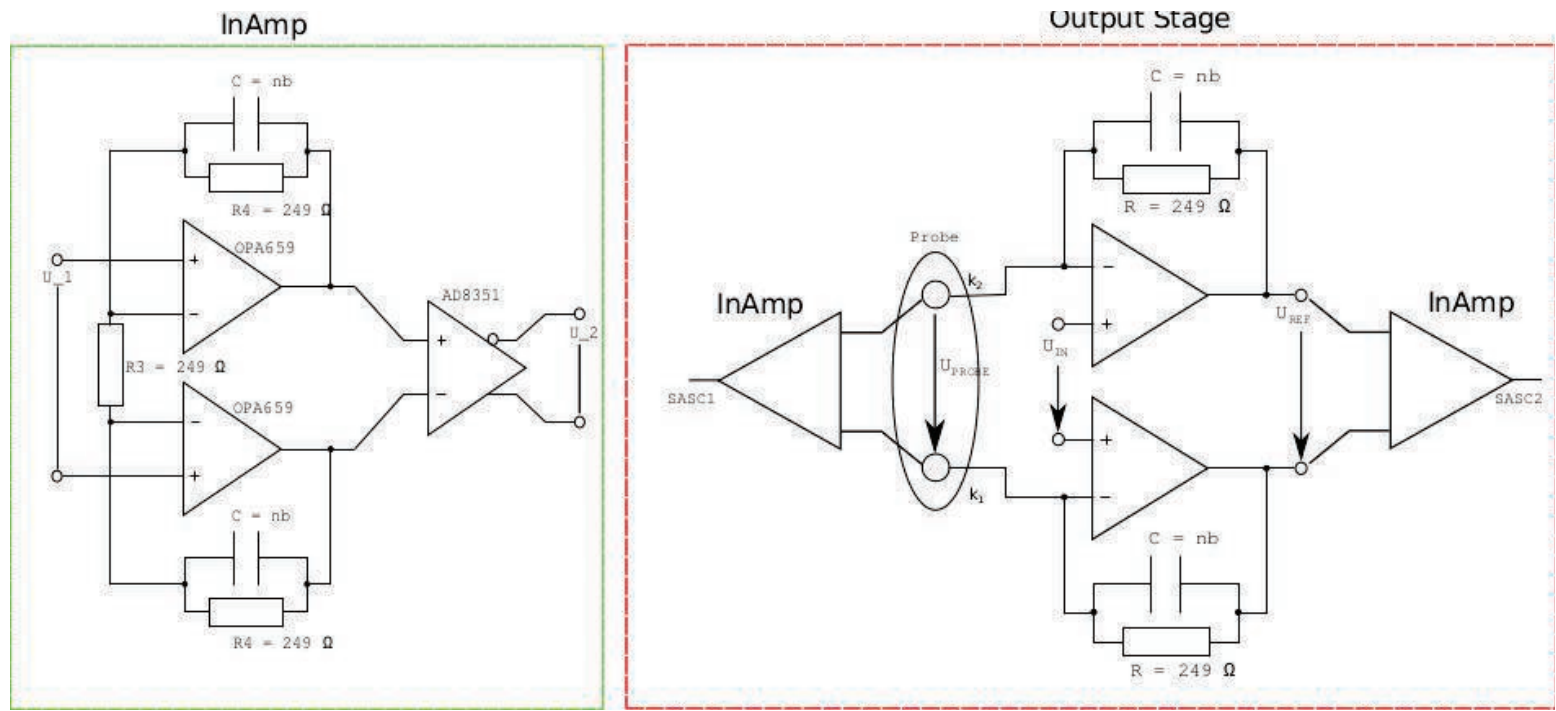

Figure 4: Output-stage (right) with details of InAmp (left) 
The IS measurement system needs a output-stage capable of driving impedance's up to $100 \mathrm{MHz}$. The architecture from Figure 3 was used for the signal-output. By the use of two non-inverting amplifier's, in a differential configuration as given in Figure 4, no external reverence for the IS measurements is needed. The impedance can be calculated based on the knowledge of the voltage $U_{\text {pobe, }} Z_{R C}$ and $U_{\text {ref }}$

$$
Z_{\text {Probe }}=\frac{U_{\text {Probe }} * 2 * Z_{R C}}{U_{\text {Ref }}-U_{\text {Probe }}}
$$

The voltages $U_{\text {pobe, }}$ and $U_{\text {ref }}$ are sampled by two instrumentation amplifier's and applied to the sample scope. The computation of the impedance will be done by an ensuring FFT, based on eq. 4 for each frequency point. The drawback of this architecture, is the dependency of the measurable impedance range, from the impedance in the feedback loop $Z_{R C}$. The gain and gain-bandwidth of the operational amplifier defines the output resistance of the structure. The op-amps should have also a high impedance at the input, to reduce the error. For this need, op-amps with FET input stages were used (OPA659 from TexasInstruments).
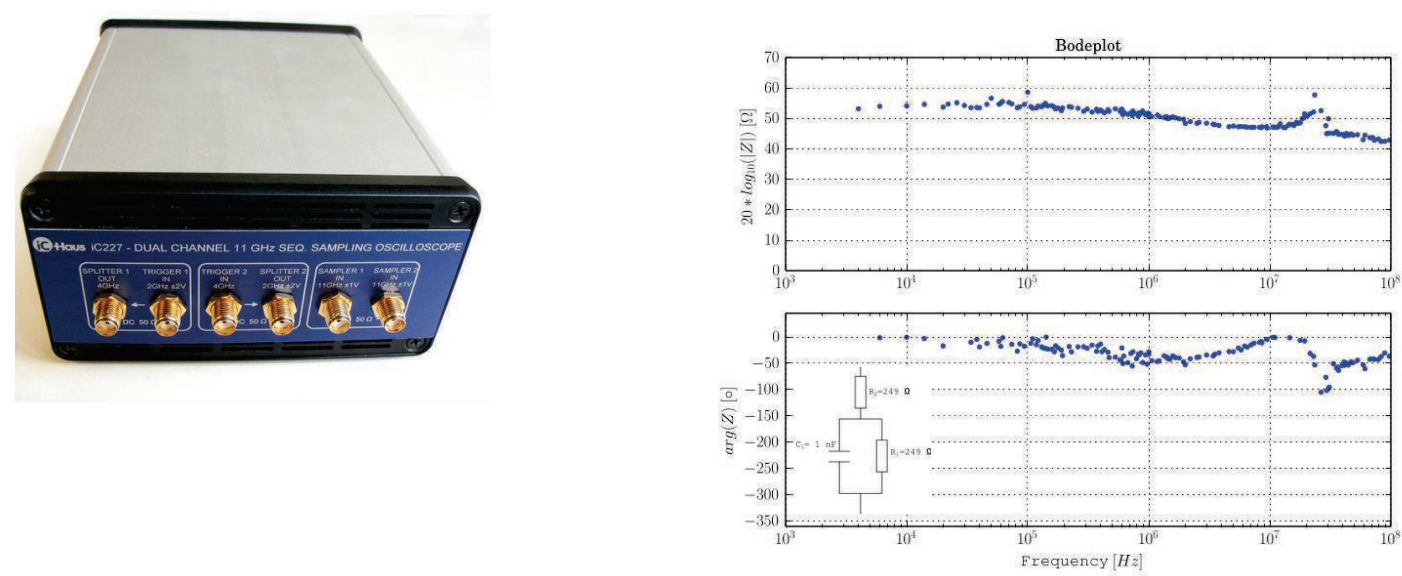

Figure 5: Picture of the proprietary sample scope (ic227) from IC-Haus

Figure 6: Measurement example from 5 $\mathrm{kHz}$ to $90 \mathrm{MHz}$ (with full system)

\section{Signal processing and measurements}

The voltages, $U_{\text {ref }}$ and $U_{\text {probe }}$ at the outputs of the InAmps, will be applied to the ic227 sample scope. The sample scope can sample synchronous two channels, without loss of the phases in between the two channels. A measurement with the sample scope takes over 11 periods of the lowest frequency in the multi-tone signal. Only time stationary are measurable with the sample scope. The sample scope sends the measured signals to a PC via USB. The results were evaluated and displayed by a Python-script.

To assess and demonstrate the current system implementation measurements with the following conditions and settings have been carried out:

- The measurements were taken by a settled system.

- A continuous multi-tone waveform was applied

- two kinds of measurements were carried out: 
- signal generation with a Tektronix AFG, to assess the capability of the outputstage up to $100 \mathrm{MHz}$

- signal-generation with the AD9102, problematic for frequency's above 10/20 MHz. Because of the hand-soldered, still quite provisional PCB. Noise and oscillationeffects disturb the measurement drastically.

- measurements starts after settling of the measurement system.

- A measurement takes over 11 periods of the lowest frequency. Only time stationary systems are measurable with the sample scope

- measured frequencies are range from $5 \mathrm{kHz}$ to $100 \mathrm{MHz}$

- Impedance range depending on the impedance in the feedback impedance in the output -stage. For the measurements the following impedance's were used.

- Lowest measured impedance were $10 \Omega$ with $50 \Omega$ in the feedback

- Highest impedance were $5 \mathrm{M} \Omega$ with $1 \mathrm{M} \Omega$ In the feedback

- usually $100 \Omega$ to $1 \mathrm{k} \Omega$ were measured with $249 \Omega$ in the feedback

\section{Measurement Results}

Common passive RLC elements were used as a DUT network for the measurements.

Also, a plate capacitance was used for the measurements, to measure the dielectric material properties.

Figure 6 shows a measurement result as example, for a measurement. A passive RC element was used as test-impedance and $249 \Omega$ were in the feedback. In Figure 6 different effects can be identified. Systematic error as result of the multi-tone signal (pattern in the frequency points). Above $10 \mathrm{MHz}$, the rising output impedance of the output stage, appears as phase-shift and attenuation of the impedance. At $10 \mathrm{MHz}$ parasitic effects appear, because the wire-lengths got in the region of $\lambda$ (14 cm wire-length).

\section{Conclusions}

This work investigated implementation options for a multi-tone sine wave impedance spectroscopy measurement system with the objective to achieve reduced measurement time along with an application-relevant bandwidth. The developed architecture was implemented in the first step as a discrete system with PCB and desktop components. Optimized multitone computation was achieved by employing a PSO algorithm for optimization. In the first experiments, a bandwidth up to $90 \mathrm{MHz}$ could be confirmed.

Future work will regard the completion of this case study, additional applications, and the consideration of monolithic integration.

\section{References}

[1] Sanchez, B.; Bragos, R.; Vandersteen, Gerd, "Influence of the multisine excitation amplitude design for biomedical applications using Impedance Spectroscopy," Engineering in Medicine and Biology

Society,EMBC, 2011 Annual International Conference of the IEEE , vol., no., pp.3975,3978, -Sept. 32011 doi: 10.1109/IEMBS.2011.6090987

[2] Bragos, R.; Blanco-Enrich, R.; Casas, O.; Rosell, J., "Characterisation of dynamic biologic systems using multisine based impedance spectroscopy," Instrumentation and Measurement Technology Conference, 2001. IMTC 2001. Proceedings of the 18th IEEE, vol.1, no., pp.44,47 vol.1, 21-23 May 2001 doi: 10.1109/IMTC.2001.928785

[3] Friese, M., "Multitone signals with low crest factor," Communications, IEEE Transactions on , vol.45, no.10, pp.1338,1344, Oct 1997,doi: 10.1109/26.634697

[4] Lingaselvan Palanichami "Multivariate Modeling And Optimization For Efficient Analog Mixed-Signal Circuit Synthesis", Master Thesis, Lehrstuhl Integrierte Sensorsysteme , TUKaiserslautern 2014 , supervisor: Prof. Dr. König , M.Sc. Abhaya Chandra Kammara

[5] ADUCM350, 16-Bit Precision, Low Power Meter on a Chip with Cortex-M3 and Connectivity, Analog-Devices; http://www.analog.com/en/products/processors-dsp/analog-microcontrollers/arm-cortex-m3processor/aducm350.htm|\#product-overview; last visted feb.2015

[6]Andrzej Lasia. Electrochemical Impedance Spectroscopy and its Applications.

Springer Verlag, 2014. 